\title{
LQAC-4: Fourth release of the Large Quasar Astrometric Catalogue
}

\section{Compilation of 443725 objects including cross-identifications with Gaia DR1 ${ }^{\star}$}

\author{
C. Gattano ${ }^{1}$, A. H. Andrei ${ }^{1,2,3}$, B. Coelho ${ }^{3}$, J. Souchay ${ }^{1}$, C. Barache ${ }^{1}$, and F. Taris ${ }^{1}$ \\ ${ }^{1}$ SYRTE, Observatoire de Paris, PSL Research University, CNRS, Sorbonne Universités, UPMC Univ. Paris 06, LNE, Paris, France \\ e-mail: cesar.gattano@u-bordeaux.fr \\ 2 Observatório Nacional/MCT, Rio de Janeiro, Brazil \\ ${ }^{3}$ Observatório do Valongo, UFRJ, Rio de Janeiro, Brazil
}

Received 26 October 2017 / Accepted 15 February 2018

\begin{abstract}
Context. From an astrometric point of view, quasars constitute the best and almost ideal reference objects in the celestial sphere, with a priori no significant proper motion. Since the third release of the Large Quasar Astrometric Catalogue (LQAC-3), a large number of quasars have been discovered, in particular those coming from the DR12Q release of the SDSS. Moreover, for cross-matched objects, we have taken advantage of the very accurate determinations of the quasars identified within the recent Gaia DR1 catalogue.

Aims. Following the same procedure as in the three previous releases of the LQAC, our aim is to compile the large majority of all the quasars recorded so far. Our goal is to record their best coordinates and substantial information concerning their physical properties such as the redshift as well as multi-bands apparent and absolute magnitudes. Emphasis is given to the results of the cross-matches with the Gaia DR1 catalogue.

Methods. New quasars coming from the DR12Q release were cross-matched with the precedent LQAC-3 compilation with a 1" search radius, in order to add the objects without counterpart to the LQAC-4 compilation. A similar cross-match was done with Gaia DR1 to identify the known quasars detected by Gaia. This enables one to improve significantly the positioning of these objects, and in parallel to study the astrometric performance of the individual catalogues of the LQAC-4 compilation. Finally, a new method was used to determine absolute magnitudes.

Results. Our final catalogue, called LQAC-4, contains 443725 objects. This is roughly $37.82 \%$ more than the number of objects recorded in the LQAC-3. Among them, 249071 were found in common with the Gaia DR1, with a 1" search radius. That corresponds to $56.13 \%$ of the whole population in the compilation.

Conclusions. The LQAC-4 delivers to the astronomical community a nearly complete catalogue of spectroscopically confirmed quasars (including a small proportion of compact AGNs), with the aim of giving their best equatorial coordinates with respect to the ICRF2 and with exhaustive additional information. For more than $50 \%$ of the sample, these coordinates come from the very recent Gaia DR1.
\end{abstract}

Key words. catalogs - astrometry - reference systems - quasars: general

\section{Introduction}

Quasars are the cornerstone of modern astrometry since they do not show any detectable proper motion or parallax thanks to their cosmological distance from the Earth. Therefore, they constitute a set of quasi-fixed directions in the universe from our Earth-centred point of view. For this property, they are the best candidates to materialize the International Celestial Reference System (ICRS; Arias et al. 1995) as a quasi-inertial reference frame, namely the ICRF, that is itself at the basis of various scientific studies in the fields of fundamental physics, geodesy, or solar system exploration. They are also of high interest for astrophysics since their extremely high luminosity is theoretically due to the presence of a massive black hole within its core. Sometimes quasars are responsible for ejections in powerful plasma

\footnotetext{
* The LQAC-4 catalog is only available at the CDS via anonymous ftp to cdsarc.u-strasbg.fr $(130.79 .128 .5)$ or via http://cdsarc.u-strasbg.fr/viz-bin/qcat?]/A+A/614/A140
}

jets that can be observed in radio from Very Long Baseline Interferometry (VLBI; Schuh \& Behrend 2012).

For all those reasons, compiled catalogues of quasars are of high value for the scientific community, and updating them on a regular basis is necessary. Historically, the first quasar catalogue was compiled by de Veny et al. (1971). They gathered 202 quasars from several observational programmes and brought a complete overview of the quasar known population at that epoch.

Following these, several studies aimed to increase the number of compiled quasars. Among them, Véron-Cetty \& Véron (2010) worked for $25 \mathrm{yr}$ from 1985 to 2010 with this same purpose and produced regularly an updated version of their compiled catalogue with the number of known quasar increasing exponentially (see Fig. 1). Their last and 13th version (Véron-Cetty \& Véron 2010), dates back to seven years ago with a complete set of 133336 quasars. Nevertheless, because of their field of interest, they stressed their production in an astrophysical point of view, at the expense of the astrometric potential of such a catalogue of extragalactic objects. 


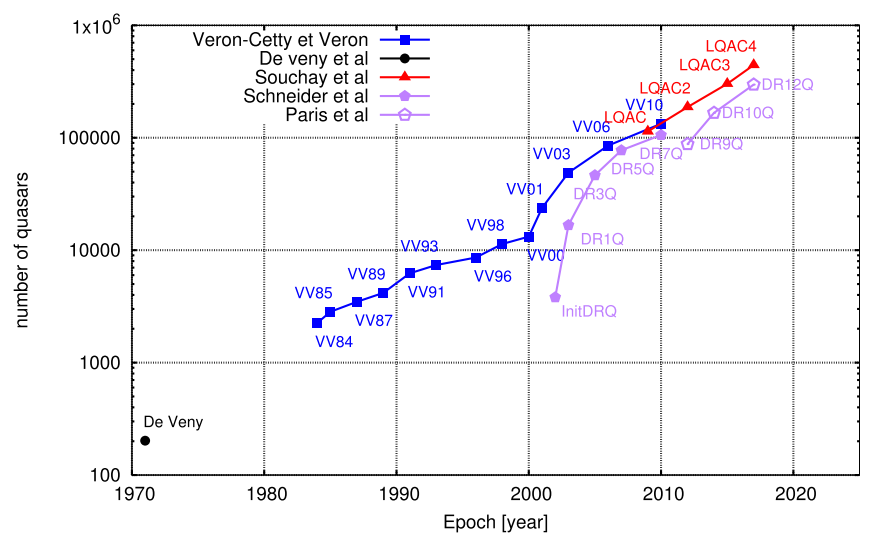

Fig. 1. Evolution of the number of objects in several quasar compiled catalogues: de Veny et al. (1971), the Véron-Cetty \& Véron (2010) catalogue, and the Large Quasar Astrometric Catalogue. We also show one of the most ambitious surveys that bring a huge number of quasars to our knowledge: the Sloan Digital Sky Survey (York et al. 2000).

To fill this gap, Souchay et al. (2009) produced their own compiled catalogue highlighting the astrometric properties of quasars, in particular by an optimized selection of celestial coordinates. This first version in Souchay et al. (2009) contained 113666 objects and the last version, called LQAC-3, gathered 321957 objects (Souchay et al. 2015). Since 2010 and the final version of the Véron-Cetty \& Véron (2010) catalogue, Souchay et al. (2009) pursued the goal of gathering the overall known quasars population from all the available surveys in a unique catalogue with both astrometric and astrophysical data.

The current version presented in this paper, called LQAC4 , falls within a new era of very huge surveys such as the first data release produced by the successful ESA astrometric space mission Gaia (GDR1; Gaia Collaboration 2016a,b), as well as the very fruitful Sloan Digital Sky Survey (SDSS), started at the beginning of the century (York et al. 2000). In Fig. 1, we display the number of quasars recorded in different versions of Véron-Cetty \& Véron (2010), of the LQAC and of the Sloan Digital Sky Survey Quasar Catalogue for comparison. We remark that since 2005, the SDSS catalogue has represented a large majority of the quasars discovered so far.

Gaia is a satellite that orbits around the Sun-Earth $L_{2}$ Lagrange point, in the opposite direction of the Sun from the Earth. The satellite is spinning at the rate of 59.' $9605 \mathrm{~s}^{-1}$. Therefore, the fixed focal plane of the telescope, shared by two different fields of view separated by a quasi-constant angle of $106.5^{\circ}$, the so-called basic angle, is continuously moving with respect to the sky and detects every crossing sources. The telescope is slowly precessing around the Sun with an angle of $45^{\circ}$ between the sun direction and the spin axis of the satellite, completing 5.8 revolutions per year. This enables it to scan the entire sky several times over the five years of planned operations.

The first data release from Gaia observations (GDR1) was produced in September 2016 (Lindegren et al. 2016) after 14 months of nominal operations. The Gaia data treatment is conceptually self-calibrated. This means that instrumental parameters are determined from the observations in parallel of science parameters. This is done through iterations of the data processing, leading each time to better results on both science and instrumental parameters. Gaia-DR1 was made available during the first step whereas the data processing, in its initial configuration, has not been totally completed, disabling the classification of the observed sources (as quasars, stars, galaxies). Consequently, among the 1141 million sources detected by Gaia, a huge number of quasars have been observed but their exact number is as yet unknown. Hence, their identification as quasars from cross-matches with quasars surveys is necessary and this constitutes a fundamental point of this paper.

In parallel, the Sloan Digital Sky Survey entered recently its fourth observation stage, called SDSS-IV/eBOSS (Dawson et al. 2016), using the $2.5 \mathrm{~m}$ Telescope located at Apache Point Observatory (Gunn et al. 2006). The previous stage, SDSS- III/BOSS (Dawson et al. 2013; Eisenstein et al. 2011), has been recently completed after five nominal years of operations. The set of data has been fully analysed, and among other results, the twelfth data release of the SDSS quasar catalogue (DR12Q) has been produced (Pâris et al. 2017). This catalogue gathers 297301 quasars scattered on $9376 \mathrm{deg}^{2}$, of which more than 200000 are new discoveries since the first two observation stage of SDSS, recorded in the seventh data release of the SDSS Quasar Catalogue (DR7Q; Schneider et al. 2010).

In the DR12Q, all quasars are automatically selected and then spectroscopically confirmed as quasars through a rigorous visual inspection. We note that the SDSS team use its own definition for a quasar: an object with a luminosity $M_{i}[z=2]<-20.5$ and either displaying at least one emission line with $F W H M>$ $500 \mathrm{~km} \mathrm{~s}^{-1}$ or, if not, having complex absorption features, such as a Lyman- $\alpha$ forest. Quasar candidates (546 856) were selected from the SDSS-III/BOSS quasar target selection (Ross et al. 2012) and ancillary programmes, from SDSS-IV/eBOSS pilot survey quasar target selection (Myers et al. 2015) and from possible quasars serendipitously targeted as galaxies with a redshift $z>2$ (Pâris et al. 2017). The main purpose of the DR12Q through the third SDSS observation stage was to have a maximized detection of quasars at $z>2.15$, with a magnitude $r \leqslant 21.85$ or $g \leqslant 22$.

In this paper we briefly present the fourth version of the LQAC compilation, named LQAC-4. In the following section, we explain how this constitutes a substantial improvement with respect to the LQAC-3, by addition of the objects coming from the DR12Q. In Sect. 3, we discuss the cross-identification of the LQAC-4 with the Gaia DR1, emphasizing the case of the SDSS. Then a study of the astrometric accuracy of individual catalogues participating to the LQAC-4 is described in Sect. 4. In Sects. 5 and 6 respectively, we present our computation of absolute magnitudes and of morphological indexes of the quasars available. We finally focus on source coordinates in Sect. 7 and, more precisely, how we selected the most accurate set of coordinates depending on the observation history of the source.

\section{The LQAC-4 update}

The LQAC-4 is constructed starting from the LQAC-3 (Souchay et al. 2015) by adding exclusively objects coming from the SDSS DR12Q release (see last section). To select a new quasar from the DR12Q not present in the LQAC-3 we adopted a $1^{\prime \prime}$ search radius limit under which two quasars are considered the same. With this threshold, a total of 121768 new objects were found. Therefore the number of objects jumps from 321957 in the LQCA-3 to 443725 objects in the LQAC-4, that constitutes a $37.82 \%$ increase. We notice that in case of cross-identification between a non SDSS quasar of the LQAC-3 and a quasar from the DR12Q, the additional useful information coming from the SDSS catalogue (as the $u, g, r, i, z$ photometry) is taken into account.

In order to get a clear look of the contribution of the new quasars from the DR12Q, we plot in Figs. 2 and 3 respectively the sky distribution of the total population of the 321957 objects 


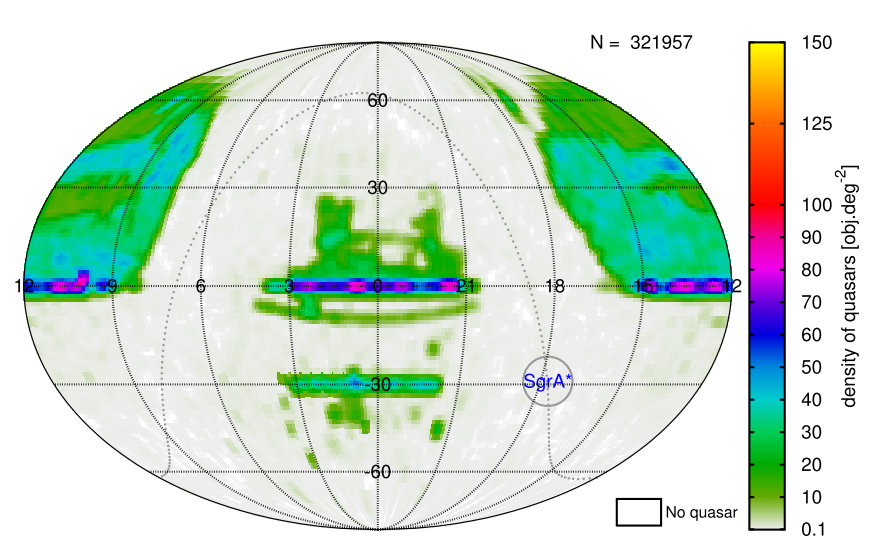

Fig. 2. Celestial sphere of the density map of quasars in the LQAC-3 in equatorial coordinates. The curved dotted line represents the galactic plane. SgrA* indicates the centre of our galaxy.

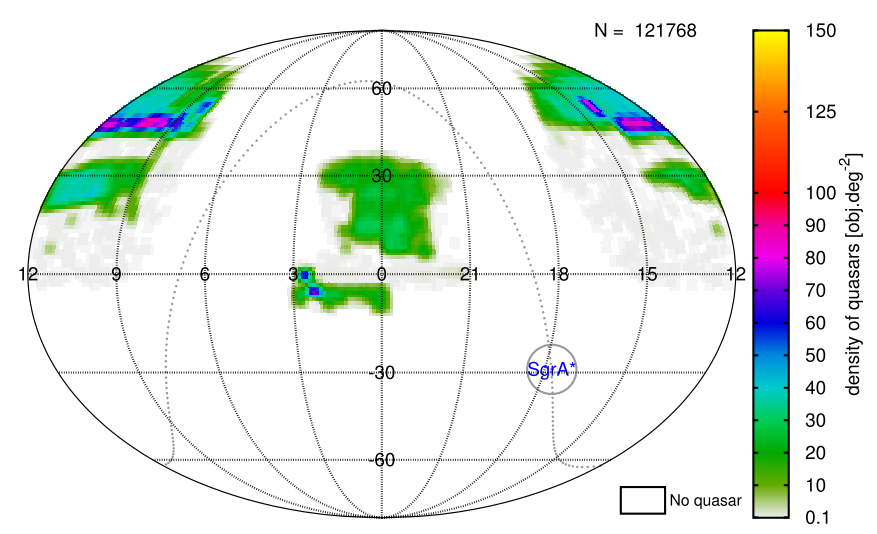

Fig. 3. Celestial sphere of the density map of new quasars (with respect to LQAC-3) in the LQAC-4 in equatorial coordinates. The curved dotted line represents the galactic plane. SgrA* indicates the centre of our galaxy.

of the LQAC-3 and of the 121768 additional quasars of the LQAC-4 coming from the DR12Q. We remark that the zones concerned by these additional quasars are globally included in those of the LQAC-3. Therefore they participate to a densification of the surface density of the sources. Moreover we plot in Figs. 4 and 5 respectively the sky distribution of the 384834 quasars of the LQAC-4 objects belonging (but not always exclusively) to the SDSS survey, and the 58891 quasars not included in that survey. We remark (Fig. 5) that objects coming from other surveys than SDSS allow us to improve the sky coverage. For example, the dense rectangular zones extending around $\left(\alpha= \pm 3 \mathrm{~h}, \delta=-30^{\circ}\right)$ and $\left(\alpha=12 \pm 3 \mathrm{~h}, \delta=0^{\circ}\right)$, with an average surface density of 30 quasars per sq. deg constitute the contribution of the 2dF Quasar Redshift Survey (Croom et al. 2004). See Souchay et al. (2012) for the exhaustive list of all the included surveys. We remark also that concerning the SDSS contribution (Fig. 4), the surface density is far from being uniform, ranging roughly from a low value at around 30 quasars per sq. deg. to a maximal density reaching 80 quasars per sq. deg inside a band located around $\delta \approx 45^{\circ}$.

\section{Identification of quasars in the Gaia DR1 catalogue}

Due to the lack of classification of objects in the Gaia DR1, no quasar could be newly discovered or confirmed with respect to

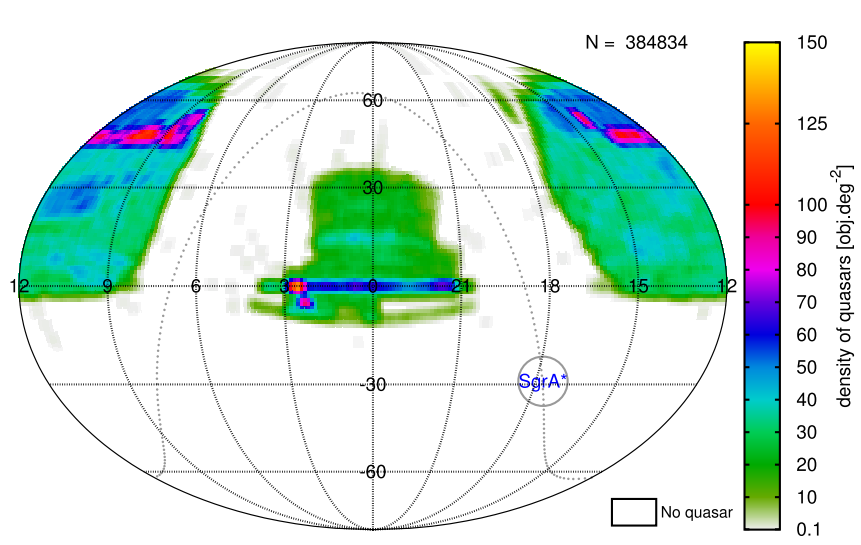

Fig. 4. Celestial sphere of the density map of quasars in the SDSS in equatorial coordinates. The curved dotted line represents the galactic plane. SgrA* indicates the centre of our galaxy.

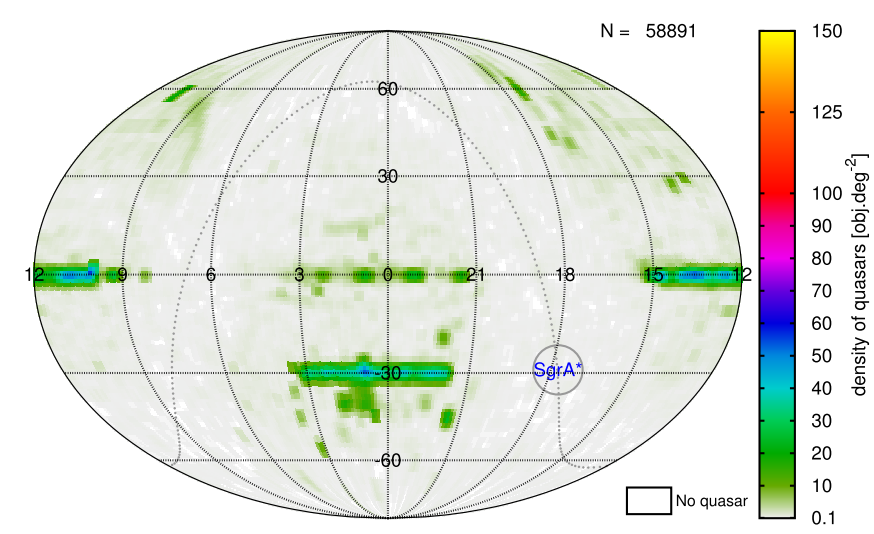

Fig. 5. Celestial sphere of the density map of quasars in the LQAC-4 but not in SDSS in equatorial coordinates. The curved dotted line represents the galactic plane. SgrA* indicates the centre of our galaxy.

a preliminary list of candidates as that given by, i.e., Secrest et al. (2015) following colour-colour selection diagrams from a huge infrared survey. For this reason, the cross-identification between a quasars compiled catalogue as the LQAC-4 and the multi-object DR1 catalogue is particularly long-awaited for several reasons. The most fundamental being that it enables us to qualify as quasars a large sample of objects among the whole DR1 population which consists of more than one billion objects. Second, for the cross-identified quasars, the DR 1 data furnishes a priori very accurate and precise celestial coordinates, at the millisecond of arc (mas) level or even one or two orders less, a considerable improvement with respect to the accuracy of the main contributor, the SDSS, typically of the order of $0.01^{\prime \prime}$ or $0.1^{\prime \prime}$. Third, the comparison of the Gaia coordinates with those determined from individual catalogues will inform directly on the astrometric quality of these catalogues. Fourth, Gaia gives additional information such as the $G$-band magnitude, in a single homogeneous photometric system.

Before investigating these various aspects in the next sections, we present in the following the results concerning the cross-identification. Fundamental questions are: how many LQAC-4 quasars are present in the Gaia DR1 release? What is the dependence of the number with respect to the search radius (quoted as $\rho$ )? What is the ratio of crossidentifications with respect to each individual catalogue? The answers to these questions are given in the following subsections. 


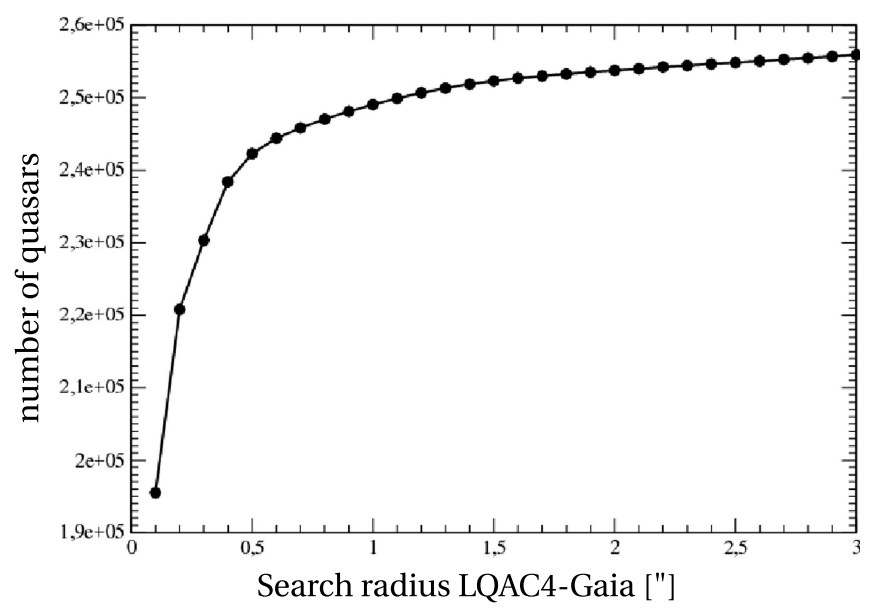

Fig. 6. Number of cross-matches between the LQAC-4 and the Gaia DR1, as a function of the search radius.

Table 1. Number of cross-matches between the LQAC-4 and the Gaia DR1 as a function of the search radius $\rho$.

\begin{tabular}{llcc}
\hline \hline $\begin{array}{l}\text { Search radius } \\
\rho\left({ }^{\prime \prime}\right)\end{array}$ & $\begin{array}{l}\text { Number of cross- } \\
\text { identifications } \\
\text { LQAC4-Gaia }\end{array}$ & $\begin{array}{c}\% \\
\text { w.r.t. } \\
\rho=1^{\prime \prime}\end{array}$ & $\begin{array}{c}\% \\
\text { w.r.t. } \\
\text { LQAC4 }\end{array}$ \\
\hline 0.1 & 195506 & 78.49 & 44.06 \\
0.2 & 220795 & 88.65 & 49.76 \\
0.3 & 230338 & 92.48 & 51.91 \\
0.4 & 238414 & 95.72 & 53.73 \\
0.5 & 242294 & 97.27 & 54.60 \\
0.6 & 244411 & 98.12 & 55.08 \\
0.7 & 245838 & 98.70 & 55.40 \\
0.8 & 247046 & 99.18 & 55.68 \\
0.9 & 248111 & 99.61 & 55.92 \\
1.0 & 249071 & 100.00 & 56.13 \\
1.2 & 250687 & 100.65 & 56.49 \\
1.4 & 251888 & 101.13 & 56.76 \\
1.6 & 252715 & 101.46 & 56.95 \\
1.8 & 253294 & 101.69 & 57.08 \\
2.0 & 253780 & 101.89 & 57.19 \\
2.2 & 254255 & 102.08 & 57.30 \\
2.4 & 254666 & 102.25 & 57.39 \\
2.6 & 255061 & 102.40 & 57.48 \\
2.8 & 255481 & 102.57 & 57.57 \\
3.0 & 255914 & 102.75 & 57.67 \\
\hline & & & \\
\hline & & & \\
\hline
\end{tabular}

Notes. The adopted threshold to admit a common identification is $\rho=1^{\prime \prime}$. The third column gives the percentage of cross-matches with respect to this threshold, the fourth one gives the percentage with respect to the whole LQAC-4 sample.

\subsection{Dependency on the search radius}

In Fig. 6 we show the dependency of the number of crossmatched objects with respect to the search radius $\rho$, and in Table 1 we present the corresponding values for each step of
Table 2. Number of quasars present in each catalogue of the LQAC-4 compilation with the number of quasars in common with the Gaia DR1 catalogue and the corresponding percentage.

\begin{tabular}{llllll}
\hline \hline $\begin{array}{l}\text { Catalogue } \\
\text { name }\end{array}$ & Flag & Nature & $\begin{array}{l}\text { No. of } \\
\text { quasars } \\
\text { (LQAC-4) }\end{array}$ & $\begin{array}{l}\text { No. of } \\
\text { quasars } \\
\text { common } \\
\text { with Gaia }\end{array}$ & Perc. \\
\hline ICRF2 & A & R & 3414 & 2314 & 67.78 \\
VLBA & B & R & 7213 & 4287 & 68.25 \\
VLA & C & R & 1858 & 1228 & 60.71 \\
JVAS & D & R & 2118 & 1373 & 64.82 \\
SDSS & E & O & 384834 & 218720 & 56.83 \\
2QZ & F & O & 23660 & 18075 & 76.39 \\
LRG & G & O & 9058 & 2474 & 27.31 \\
FIRST & H & O & 969 & 909 & 93.80 \\
HB & I & O \& R & 6720 & 5991 & 89.15 \\
\hline
\end{tabular}

Notes. "O" and "R" are used respectively for optical and radio catalogue.

$\rho$. Our conventionally adopted threshold for validating the identifications between catalogues is set at $\rho=1^{\prime \prime}$ taken from the LQAC position of the source. This threshold looks reasonable, as given the astrometric precision of individual catalogues generally significantly smaller (see Souchay et al. 2015) and given the small slope of the curve after this value (Fig. 6). We notice that no double identification was found. At this threshold we find 249071 objects in common, that represents $56.13 \%$ of the total LQAC-4 sample. Then the number of new cross-matches slightly increases after the threshold above. For instance 3248 new crossmatches occur in the interval $1^{\prime \prime}<\rho<1.5^{\prime \prime}$. Further investigation including visual inspection is needed to characterize objects matched at these larger separations.

\subsection{Cross-matches of individual catalogues with Gaia DR1}

As mentioned above the LQAC-4 catalogue is a compilation of 443725 objects duly identified as quasars or point-like AGNs. Any of them is accompanied by several flags (from A to I) indicating their presence in each catalogue participating to the compilation. See Table 2 and LQAC-3 publication (Souchay et al. 2015) for corresponding catalogues and their characteristics.

This procedure allows us to carry out easily a completeness study consisting of determining the percentage of quasars of each catalogue present in the DR1 release, still by adopting the search radius $\rho=1^{\prime \prime}$. In Table 2, we show the corresponding results. We notice that for the four VLBI catalogues (ICRF2, VLBA, VLA, JVAS) the percentage ranges between $64 \%$ and $68 \%$. It exhibits two extreme values respectively for the LRG catalogue $(27.31 \%)$ and for the FIRST one (93.8\%).

\subsection{Specific case of the cross-matches of the SDSS catalogue with Gaia DR1}

The case of the cross-identification of the SDSS survey with the DR1 looks particularly interesting. This is firstly because of the relative high surface density of quasars it contains in the zone 


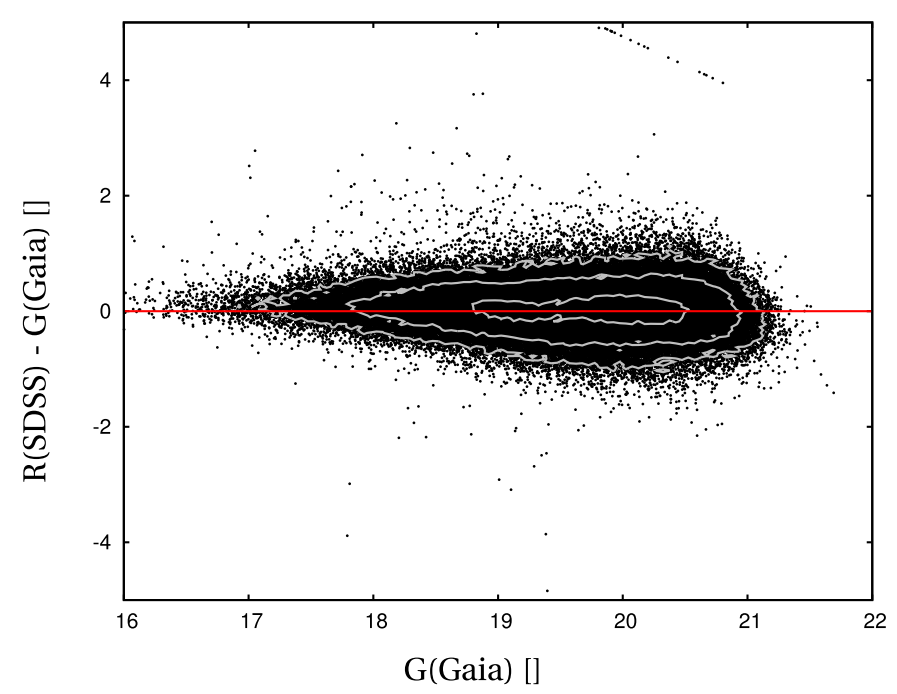

Fig. 7. Magnitude difference $r$ (SDSS)-G (Gaia) for the quasars in common in the two surveys. Contour-plots are added to estimate the number of points in the saturated area. The largest, intermediate, and shortest contour-plots correspond respectively to, ten points, 50 points, and 250 points in a pixel of 0.05 magnitude $\times 0.05$ magnitude.

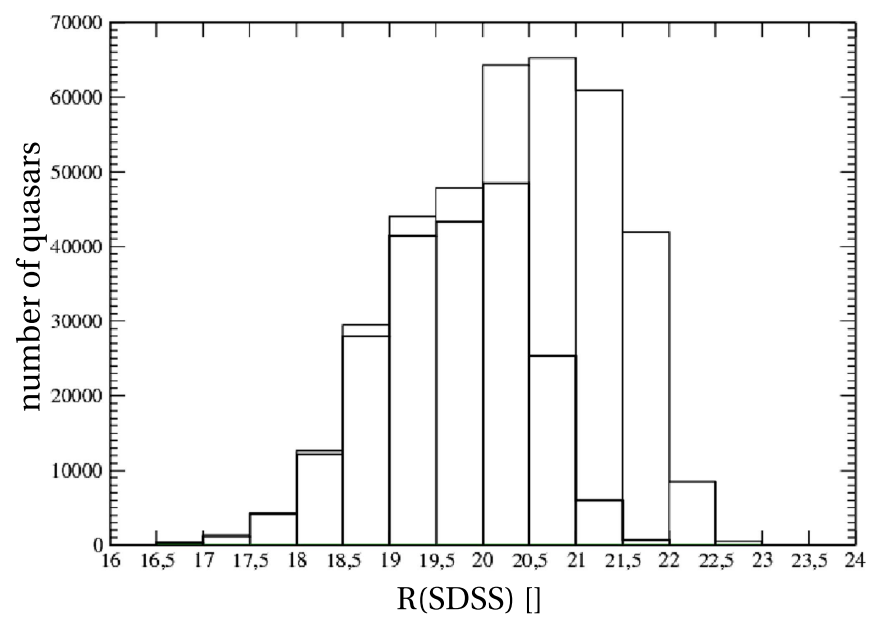

Fig. 8. Histogram of the number of SDSS quasars per bin of $r$ (SDSS). In bold we plot the number of quasars reckoned by cross identification with Gaia-DR1.

explored, corresponding roughly to one fourth of the celestial sphere, and secondly because the upper magnitude threshold of the SDSS is a priori quite above the detection threshold of Gaia.

We count 218720 SDSS objects that are matched with a Gaia entry within $\rho=1^{\prime \prime}$, that corresponds to $56.83 \%$ of the whole SDSS quasar sample. For a detailed study of the comparison of the Gaia broad-band photometry and the SDSS normalized passband photometry, we can refer to Jordi et al. (2010). As mentioned by the authors, the Gaia $G$ band has a central wavelength at $\lambda_{0}=673 \mathrm{~nm}$ and a FWHM $\Delta \lambda=440 \mathrm{~nm}$, whereas the SDSS $r$ band corresponds to $\lambda_{0}=620 \mathrm{~nm}$ and a FWHM $\Delta \lambda=113 \mathrm{~nm}$. Thus we can remark that the central wavelengths are very close, and despite the big difference in bandwidth, we can expect a good correspondence between the two magnitude determinations for the quasars in common. This is illustrated in Fig. 7 where we can observe a good agreement between the $G$ (Gaia) and the $r$ (SDSS) values of generally better than \pm 1 mag. with a standard deviation increasing steadily due to statistical effect as the number of quasars increases between $G=16$ and $G=21.5$, as shown by the grey contour-plots. We notice that the difference of value can be explained by the uncertainty of each measurement. This uncertainty generally increases with the magnitude, by the difference in the characteristics of the photometry as indicated above and by the fact that the quasars present significant flux variations at the level of $0.1 \mathrm{mag}$. with time scales of the order of weeks or months, in accordance with the time delay between the measurements from SDSS and Gaia respectively.

In Fig. 8, we show the histogram of the number of quasars in common in SDSS and in Gaia DR1 in comparison with the total number of SDSS objects, with respect to each $r$ magnitude bin. The results match the expectations, that a large majority of SDSS quasars are reckoned for $r<20$, but the percentage diminishes significantly for $20<r<20.5$. It becomes smaller than $50 \%$ for $20.5<r<21$ and is very small beyond $r=21$. Here also, the flux variability of the objects and the difference of the photometric characteristics between the Gaia $G$ band and the SDSS $r$ band can both explain the lack of completeness for $20.5<r<21$, as well as the small signal-to-noise ratio in the detection, taking into account that the Gaia threshold for $G$ is close to $G=21$.

\section{Astrometric accuracy of individual catalogues}

The coordinates of the objects in the GDR1 catalogue have an outstanding accuracy at the level of 0.1 mas or 0.01 mas. This accuracy enables us to estimate the astrometric quality of any individual catalogue of the LQAC-4 from their cross-identification with the GDR 1 catalogue. To this end, we used direct determination of the difference of coordinates.

\subsection{Coordinates differences between catalogues and Gaia DR1}

A similar determination of coordinates differences is done in, for instance Fig. 9, which shows the histograms of the distribution of the differences in right ascension and declination between the ICRF2 and the GDR1 catalogues. We can observe that the majority of values range in the interval \pm 5 mas in both histograms, which are similar to those presented in a more detailed study by Mignard et al. (2016). We notice that ICRF2 positions are determined from VLBI radio observations, and that the link between the VLBI radiocentres and the Gaia photocentres was subject to recent deep investigations (Kovalev et al. 2017; Petrov \& Kovalev 2017 a,b). Nevertheless we remark that the quality of the ICRF2Gaia link is affected by a few number of outliers, as shown in the bi-dimensional plot in Fig. 10 where differences at the level of several 100 mas are found, which are far larger than the formal positional uncertainty.

The same determination of differences between coordinates is done for the SDSS, for which the offsets with respect to Gaia DR1 in right ascension and declination are shown in Fig. 11. In both graphs the Gaussian feature of the histograms shows up clearly with a majority of values ranging within the interval \pm 200 mas, which is 20 times higher than in the precedent case of the ICRF2. In addition we can easily see an anomaly characterized by a secondary peak in declination (Fig. 12), with an excess of objects gathering around $\Delta \delta \approx 300$ mas.

In order to clarify the origin of this anomaly we plot in Fig. 13 the two-dimensional positioning of the differences in equatorial coordinates. This plot shows neatly that the outliers to a large extend belong to the new quasars of the LQAC-4: those coming from the DR12Q release of the SDSS. They are concentrated around the point with values $\Delta \alpha \cos \delta=-80$ mas; 

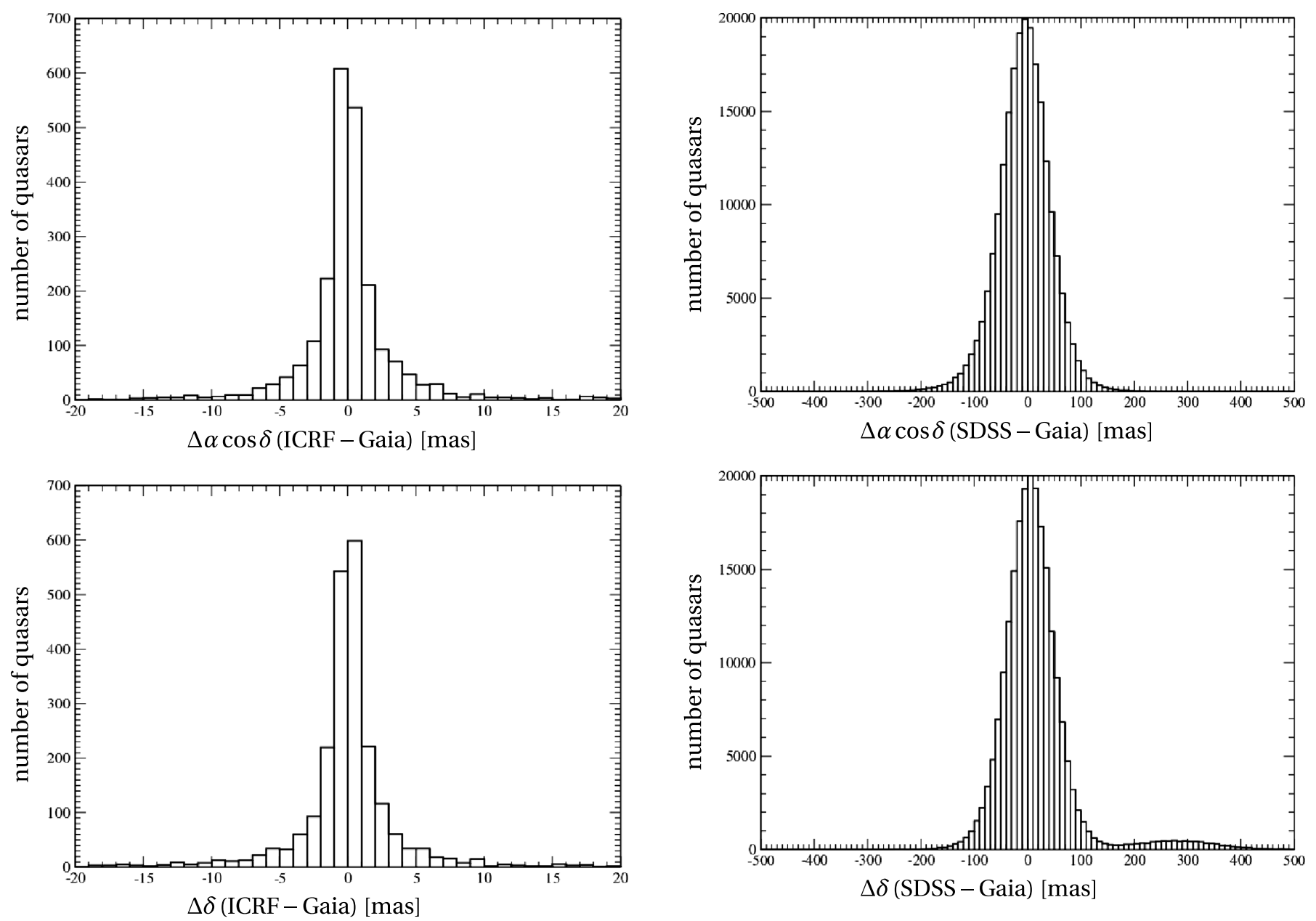

Fig. 9. Distribution of the differences in right ascension (top) and in declination (bottom) between the ICRF2 and the Gaia positions, for the quasars with values within \pm 20 mas.

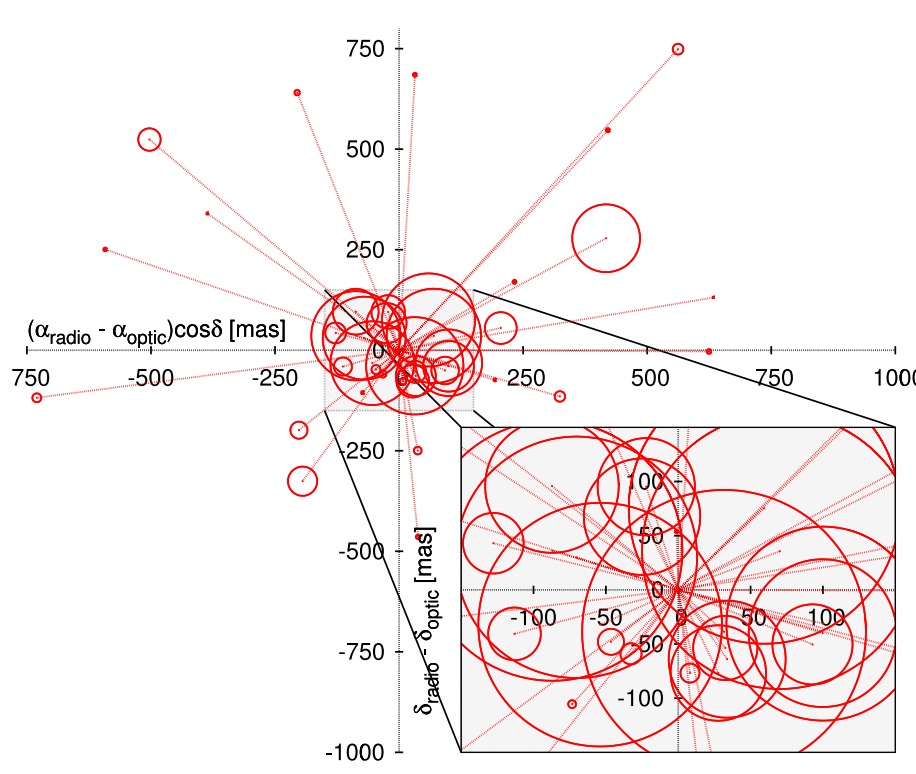

Fig. 10. Two-dimensional plot $\Delta \delta$ vs. $\Delta \alpha \cos \delta$ of the differences between the VLBI radio positions ICRF2 and the optical Gaia positions for the quasars with angular offset larger than 50 mas. Circles stand for the combined error of the two measurements.

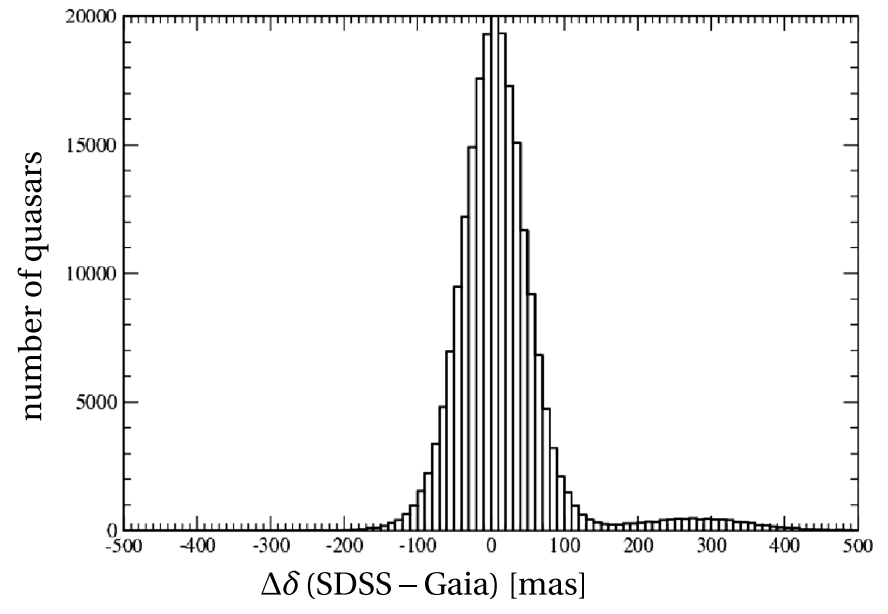

Fig. 11. Distribution of the differences in right ascension (top) and in declination (bottom) between the SDSS and the Gaia positions, limited to the quasars with values within \pm 500 mas.

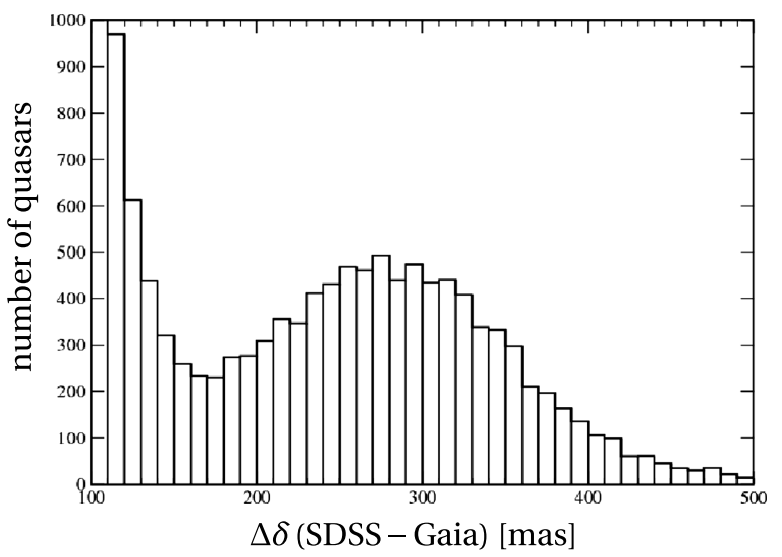

Fig. 12. Distribution of the differences in declination between the SDSS and the Gaia positions, limited to the quasars with values between 100 mas and 500 mas.

$\Delta \delta=300$ mas. This supposes the presence of a specific problem in the astrometric reduction pipeline for quasars of the DR12Q release. This problem should concern a well defined area of the sky. To confirm this hypothesis we plot in Fig. 14 the spatial distribution of the outliers detected in Fig. 12, in the celestial sphere. Indeed, that procedure indicates clearly that the outliers are occupying a very well delimited zone north of $\delta=46^{\circ}$. A magnification of the zone in Fig. 15 shows that the outliers 


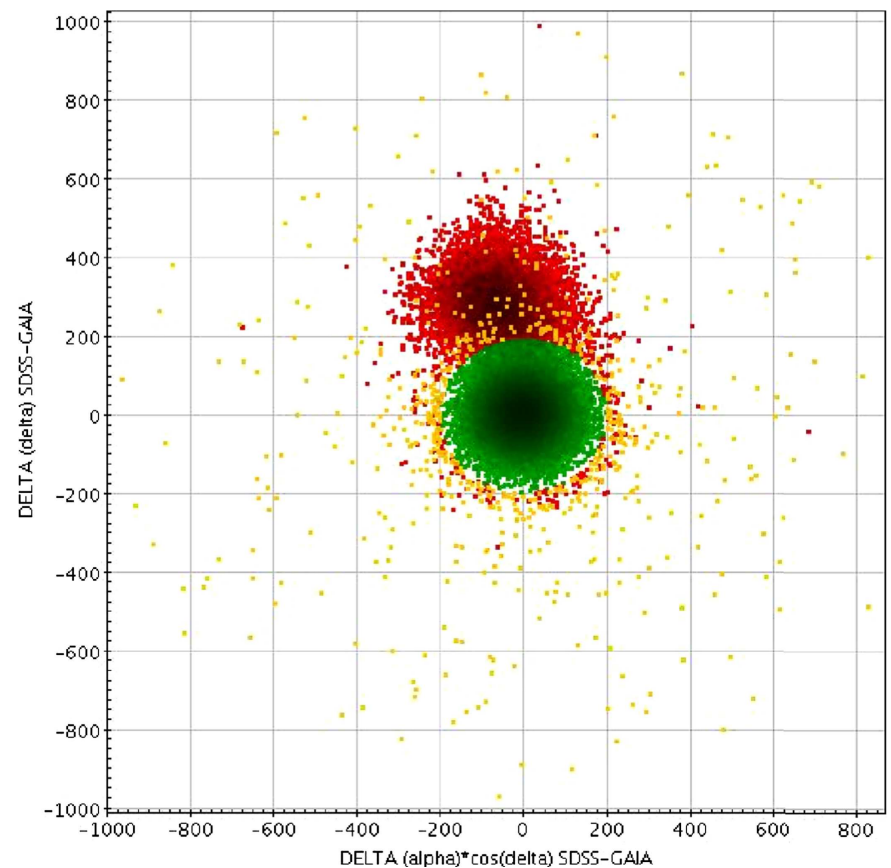

Fig. 13. Two-dimensional plot of the offsets $\Delta \delta$ vs. $\Delta \alpha \cos \delta$ of the SDSS with respect to the Gaia DR1 positions. In blue we plot the SDSS quasars for which $\rho<200$ mas, belonging to the LQAC-3; in green, the SDSS quasars for which $\rho<200$ mas, belonging to the DR12Q (LQAC4-LQAC-3); in yellow, the quasars with $\rho>200$ mas belonging to the LQAC-3; in red, the SDSS quasars with $\rho>200$ mas belonging to the DR12Q (LQAC-4-LQAC-3).

are mixed homogeneously with quasars already present in the LQAC-3, with offsets of smaller than 200 mas.

To illustrate what the second most important catalogue of the compilation in terms of number of objects, the $2 \mathrm{dF}$ QSO Redshift Survey (2QZ; Croom et al. 2004) looks like with respect to Gaia-DR1, we plot in Fig. 16 the histograms of equatorial coordinates differences (like Figs. 9 and 11 for respectively ICRF and SDSS). We see that the Gaussian features are less regular and that the coverage is more extended (between \pm 800 mas), illustrating a precision weaker than that for the SDSS. We also see that histograms do not peak at zero but are slightly shifted to negative values. This could be an effect of the different orientations of the two catalogues. We explore that question in Sect. 4.2 .

In Table 3, we show the mean and standard deviation in right ascension and declination for the coordinates differences between the Gaia values and those from each catalogue. Attention must be drawn to the fact that for each catalogue we retain only the objects which deliver a priori the best set of coordinates for the LQAC-4 according to a rule, based on the flags (A to I) already established in the previous updates of the LQAC (Souchay et al. 2015). We notice that the values $\sigma_{\alpha \cos \delta}=37.56$ mas and $\sigma_{\delta}=35.49$ mas for the GDR1-ICRF2 computations look rather big in comparison with the differences exhibited in Fig. 9. This is, indeed, due to the presence of large number of outliers as shown in Fig. 10. We remark that the best values of $\sigma_{\alpha \cos \delta}$ and $\sigma_{\delta}$ are obtained for the JVAS sample (flag D), but with a comparatively small set of 105 objects, which are naturally not present in ICRF2, VLBA, and VLA catalogues (flags A, B, and C), and without outliers. The comparison of SDSS (flag E) with GDR1 is characterized by differences spread on 0 .' $^{\prime} 052$ in right ascension and 0.'074 in declination, whereas the 2QZ (flag F) gives significantly worse values (respectively

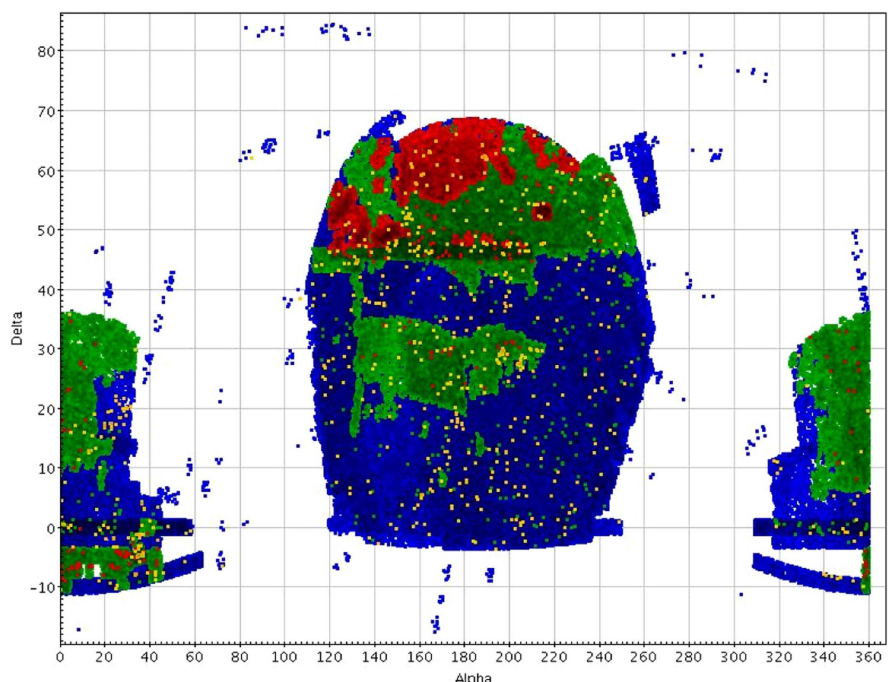

Fig. 14. Equatorial coordinates of the SDSS quasars plotted in Fig. 13. The colour codes of the quasars remain the same.

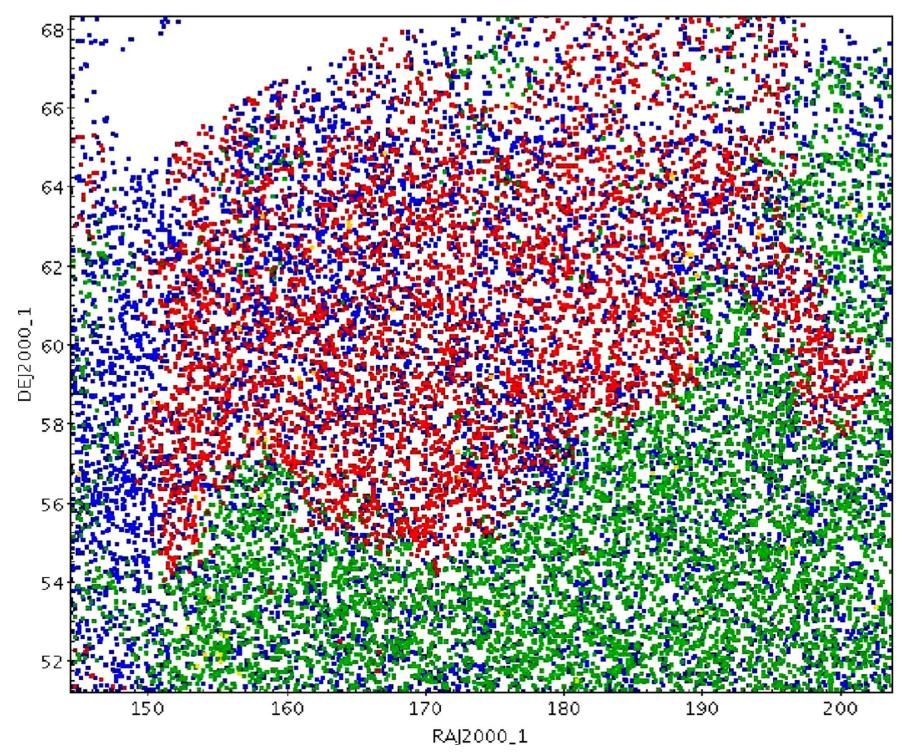

Fig. 15. Magnification of Fig. 14 above.

0.228 and 0.243$)$. Finally, the mean offset on both coordinates (cols. 5 and 6) for those optical catalogues are also significant with respect to the standard errors given in cols. 9 and 10 , whereas for radio catalogues (A, B, C, and D), they are not. Rotation between catalogues can explain this fact, as seen in the following.

\subsection{Rotations between catalogues and GDR1}

As its predecessors, the LQAC-4 is constructed with the aim of giving the best determination of celestial coordinates, based on the astrometric performance of individual catalogues. The flags displayed in Table 3 corresponds to a hierarchy, which privileges the coordinates of the catalogue of a given flag (ICRF2 for flag A, VLBA for flag B etc.) with respect to the following ones, when these coordinates are available in both catalogues.

Starting from the cross-identification between the LQAC and Gaia, we also computed the global difference in orientation between each individual catalogue and Gaia. This difference 

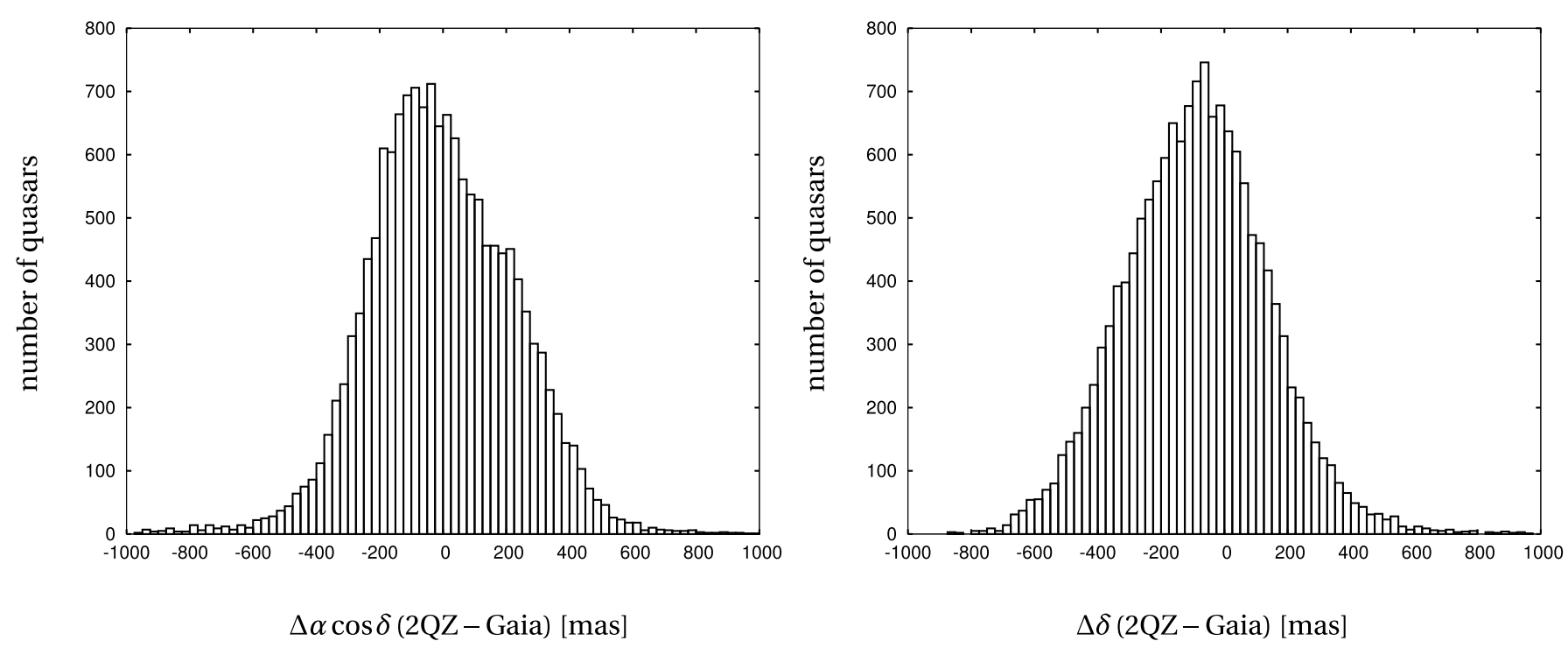

Fig. 16. Distribution of the differences in right ascension (top) and in declination (bottom) between the 2QZ and the Gaia positions, limited to the quasars with values within \pm 1000 mas.

Table 3. Summary of the LQAC-4 compilation and the cross-identification with the Gaia-DR1 catalogue.

\begin{tabular}{llllllllll}
\hline \hline $\begin{array}{l}\text { Catalogue } \\
\text { name }\end{array}$ & $\begin{array}{l}\text { No. of } \\
\text { quasars }\end{array}$ & $\begin{array}{l}\text { No. of } \\
\text { quasars } \\
\text { taken } \\
\text { for } \\
\text { coord. }\end{array}$ & $\begin{array}{l}\text { No. of } \\
\text { quasars } \\
\text { cross-id } \\
\text { with } \\
\text { Gaia }\end{array}$ & $\begin{array}{l}\langle\Delta \alpha \cos \delta\rangle \\
\text { (mas) }\end{array}$ & $\begin{array}{l}\langle\Delta \delta\rangle \\
\text { (mas) }\end{array}$ & $\begin{array}{l}\sigma_{\alpha \cos \delta} \\
\text { (mas) }\end{array}$ & $\begin{array}{l}\sigma_{\delta} \\
\text { (mas) }\end{array}$ & $\begin{array}{l}\sigma_{\langle\Delta \alpha \cos \delta\rangle} \\
\text { (mas) }\end{array}$ & $\begin{array}{l}\sigma_{\langle\Delta \delta\rangle} \\
\text { (mas) }\end{array}$ \\
\hline ICRF2 (A) & 3414 & 3414 & 2314 & 0.26 & 1.24 & 37.56 & 35.49 & 0.78 & 0.74 \\
VLBA (B) & 7213 & 3800 & 1973 & -0.01 & 0.84 & 66.90 & 55.90 & 1.51 & 1.26 \\
VLA (C) & 1858 & 151 & 30 & 19.35 & 24.27 & 247.90 & 278.70 & 45.26 & 50.88 \\
JVAS (D) & 2118 & 255 & 105 & 6.75 & 2.85 & 16.61 & 28.40 & 1.62 & 2.77 \\
SDSS (E) & 384834 & 383742 & 217748 & -6.39 & -15.48 & 51.56 & 73.70 & 0.11 & 0.16 \\
2QZ (F) & 23660 & 20355 & 15283 & 5.66 & 86.79 & 227.83 & 226.75 & 1.84 & 1.83 \\
LRG (G) & 9058 & 6528 & 1258 & 7.81 & -19.12 & 53.32 & 47.78 & 1.50 & 1.34 \\
FIRST (H) & 969 & 131 & 105 & 39.48 & 0.81 & 252.06 & 326.79 & 24.60 & 31.89 \\
HB (I) & 6720 & 3659 & 3093 & 85.38 & -128.82 & 245.75 & 241.62 & 4.42 & 4.34 \\
\hline
\end{tabular}

Notes. The table includes: number of quasars for each catalogue of the LQAC-4 compilation (Col. 2); number of quasars with the most accurate equatorial coordinates taken from the corresponding catalogue of the line (Col. 3); number of objects cross-identified with Gaia (Col. 4); mean for the difference of equatorial coordinates (Cols. 5 and 6); standard deviation for the difference of equatorial coordinates (Cols. 7 and 8); standard deviation for the difference mean (Cols. 9 and 10).

is quantified by three angles of rotation that enable us to align the celestial frame defined by the coordinates of the sources of the chosen catalogue observed by Gaia onto the celestial frame defined by the coordinates of these same sources taken in the Gaia catalogue. Results are given in Table 4.

Because of the hierarchy within the LQAC to obtain the position of a source (catalogue A has priority over B, B over C, etc.), we proceeded iteratively to compute the orientation of all catalogues. First, we selected sources in catalogue A (namely ICRF2) and separated them from the remaining population of the LQAC. Then, we kept the objects detected by Gaia and retrieved their two sets of positions (from Gaia and from catalogue A). From the differences, we adjusted using the least squares method the angles of rotation $A_{x}, A_{y}$, and $A_{z}$ given in the model:

$$
\begin{aligned}
& \alpha-\alpha_{G}=A_{x} \tan \delta_{G} \cos \alpha_{G}+A_{y} \tan \delta_{G} \sin \alpha_{G}-A_{3} \\
& \delta-\delta_{G}=-A_{x} \sin \alpha_{G}+A_{y} \cos \alpha_{G} .
\end{aligned}
$$

Then we proceeded again with the remaining population of the LQAC by selecting sources in catalogue B (VLBA), and so on. The least-squares fitting takes into account the uncertainties of the positions when they are available, otherwise a general statistical error for each catalogue was computed beforehand as the standard deviation of the catalogue position with respect to the Gaia position.

First of all, we can see in Table 4 that the largest differences in orientation correspond to catalogues with the worst astrometry, for example catalogues F, H, and I, which need an alignment of more than 50 mas. Second, for all the optical catalogues (catalogue $\mathrm{E}$ and after), the differences in orientation are significant: they exceed $3 \sigma$, i.e. three times the formal error given by the least squares adjustment. Finally, we can see that the orientation is not statistically significant for the radio catalogues A, B, C, and D, especially for the first one which corresponds to ICRF2. This was expected because the Gaia catalogue has been aligned onto the latter (Lindegren et al. 2016). 
Table 4. Orientation differences between each catalogue and Gaia.

\begin{tabular}{rrrrrrrrrrrr}
\hline \hline$A_{x}$ & $\begin{array}{r}\text { Value } \\
{[\mathrm{mas}]}\end{array}$ & $\begin{array}{r}\text { Error } \sigma \\
{[\mathrm{mas}]}\end{array}$ & $\begin{array}{r}\text { Value } \\
{[\sigma]}\end{array}$ & $A_{y}$ & $\begin{array}{r}\text { Value } \\
{[\mathrm{mas}]}\end{array}$ & $\begin{array}{r}\text { Error } \sigma \\
{[\mathrm{mas}]}\end{array}$ & $\begin{array}{r}\text { Value } \\
{[\sigma]}\end{array}$ & $\begin{array}{r}A_{z} \\
\text { Value } \\
{[\mathrm{mas}]}\end{array}$ & $\begin{array}{r}\text { Error } \sigma \\
{[\mathrm{mas}]}\end{array}$ & $\begin{array}{r}\text { Value } \\
{[\sigma]}\end{array}$ & $\begin{array}{r}\text { No. of } \\
\text { sources }\end{array}$ \\
\hline $\mathrm{A}$ & 0.2 & 0.5 & 0.4 & -0.1 & 0.4 & -0.4 & 0.5 & 0.5 & 1.0 & 2314 \\
$\mathrm{~B}$ & 1.4 & 1.7 & 0.8 & -0.2 & 1.6 & -0.1 & 4.1 & 1.8 & 2.3 & 1973 \\
$\mathrm{C}$ & -18.7 & 10.3 & -1.8 & -16.9 & 12.4 & -1.4 & 8.4 & 8.7 & 1.0 & 30 \\
$\mathrm{D}$ & 2.7 & 4.1 & 0.7 & 2.5 & 3.9 & 0.6 & 6.3 & 3.0 & 2.1 & 105 \\
$\mathrm{E}$ & 7.0 & 0.3 & 21.3 & 14.4 & 0.3 & 47.3 & -7.3 & 0.2 & -29.4 & 217748 \\
$\mathrm{~F}$ & -81.0 & 5.3 & -15.3 & 71.6 & 2.8 & 26.0 & -6.7 & 2.4 & -2.8 & 15283 \\
$\mathrm{G}$ & 6.0 & 3.0 & 2.0 & -17.2 & 1.9 & -8.9 & 0.8 & 1.7 & 0.4 & 1258 \\
$\mathrm{H}$ & -124.5 & 47.8 & -2.6 & -4.7 & 42.2 & -0.1 & 57.6 & 38.4 & 1.5 & 105 \\
$\mathrm{I}$ & -3.8 & 6.2 & -0.6 & -40.5 & 4.7 & -8.6 & -44.6 & 5.7 & -7.9 & 3093 \\
\hline
\end{tabular}

Notes. A rotation parametrized by these three angles aligns the catalogue on Gaia: $A_{x}$ is the angle of rotation around the $(O x)$ axis of the corresponding Gaia frame, $A_{y}$ is the angle around $(O y)$, and $A_{z}$ the angle around $(O z) . \sigma$ are the formal errors. We provide normalized values of each parameter to its formal error

\section{Computation of absolute magnitudes}

Among the additional data in the LQAC that complete the information gathered from the original catalogues we give the absolute magnitudes of the quasars in the five SDSS bands $(u, g$, $r, i$ and $z$ ), when it is possible. We note that the absolute magnitude quantifies the intrinsic luminosity of celestial objects, for example quasars in the context of this work, considering a given wavelength band. Consequently, absolute magnitudes are supposed to be unaffected by the cosmological redshift due to the expansion of the Universe.

For this purpose we applied a new method of computation developed recently by one of the authors (Coelho et al. 2017). With that method, absolute magnitudes are calculated with the following equation:

$M=m+5-5 \log \left(D_{L}\right)-A-A_{\mathrm{IG}}-A_{\text {host }}-K=M^{*}-K$,

where $m$ refers to the apparent magnitude of the quasar corresponding to the given bandwidth and $D_{L}$ is the luminosity distance determined according to Hogg (2000). The four components $A, A_{\mathrm{IG}}, A_{\text {host, }}$ and $K$ account for corrections which characterize effects that can significantly affect the result if they are not considered in the calculation.

The first component, $A$, corrects the Galactic extinction due to dust of the Milky Way present on the quasar line of sight. Apparent SDSS magnitudes were therefore corrected from that effect using the classical maps of Schlegel et al. (1998).

The second component, $A_{\mathrm{IG}}$, corrects the intergalactic (IG) light absorption associated with the Lyman $\alpha(\operatorname{Ly} \alpha)$ forest. More precisely, it consists essentially of Ly $\alpha$ (1215.67 $\AA$ ) resonance absorption lines in different redshifts (Lynds 1971), produced when the QSO's light passes through intergalactic clouds of neutral hydrogen (see Rauch 1998, and references therein). The corresponding corrections were extracted from the Type $\mathrm{I}^{1}$ quasi-stellar object (QSO) curves of Meiksin (2006).

The third correction, $A_{\text {host }}$, is relative to the quasar reddening caused by the dust in its host galaxy. The Small Magellanic Cloud "bar" (SMC "bar") extinction law has been validated as the best description to describe the reddening of large quasar populations (Hopkins et al. 2004; Krawczyk et al. 2015; Coelho

\footnotetext{
1 Type I QSO refers to active galactic nuclei that exhibit broad and narrow emission lines and are interpreted to be seen quite away from the torus plan.
}

et al. 2017). Thus, for the computation of this correction we considered the SMC bar extinction law from Gordon et al. (2003).

Finally, the absolute magnitude was obtained by taking into account a last correction, the so-called K-correction which represents the redshift effect on the radiation observed in each bandpass, due to the expansion of the Universe.

The main difference between our approach to obtain the absolute magnitudes and the precedent ones is the use of the Gaia library of synthetic spectra (Claeskens et al. 2006) to model the quasar spectral energy distribution (SED). This library allows us to use models with different intensities for the emission lines, and different values of spectral index for the continuum. The selection is made by minimizing the differences between the colours from the selected synthetic spectrum and the colours $M_{u}^{*}-M_{i}^{*}, M_{g}^{*}-M_{i}^{*}, M_{r}^{*}-M_{i}^{*}$, and $M_{i}^{*}-M_{z}^{*}$. With this process we obtained the scaling factor which makes it possible to use the synthetic spectrum selected to compute the absolute magnitude $M$ for each object.

This method represents a more complete approach compared to previous ones used in the literature. It gives significant differences in the results for high redshifts, as demonstrated by Coelho et al. (2017).

\section{Studies of morphology}

Mapping of quasars through VLBI is a major method to understand their inhomogeneous, dense, and turbulent surrounding medium. Structures such as the narrow and broad line regions, as well as density fluctuations in the inner and extra galactic space can thus be modelled (Petrov \& Kovalev 2017b). Conversely, the most interesting maps astrophysics-wise correspond to the sources that are less appropriate astrometry-wise (Finet \& Surdej 2016). In the optical band the current achievable resolution is at least 100 times worse than in VLBI, nonetheless morphology information is just as important (Michalik \& Lindegren 2016; Bachchan et al. 2016).

A large proportion of the results from the Gaia mission lie over the built-in astrometric catalogue. It will considerably densify the Celestial Reference Frame constructed from quasars observed during the mission, namely the GCRF (Gaia Celestial Reference Frame, Andrei et al. 2012c). For this purpose, Gaia quasars astrometry may be impacted if the objects show extended morphology (Hobbs et al. 2017), either true departures (in a 


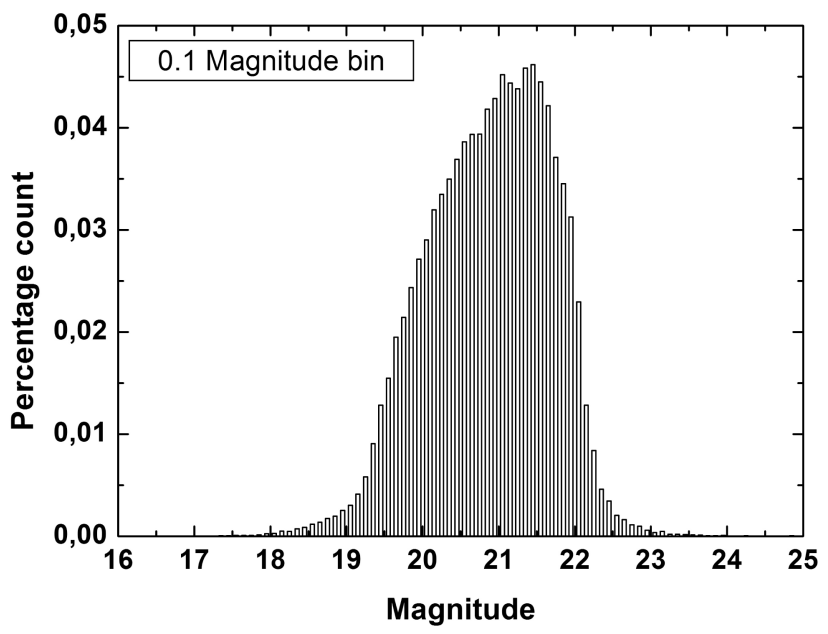

Fig. 17. Magnitude distribution histogram for the 121461 new quasars in the LQAC4 for which DSS images and the morphological indexes were searched for and calculated. The percentage counts are obtained in 0.1 magnitude bins. The magnitudes are nearly all $\mathrm{R}$, or otherwise in the closer optical band available. The average magnitude is 20.86 , and the mode is 21.45 , which values are as those of the LQAC3 version. No dependence of the morphological indexes with magnitude is found.

few cases) of a very compact one, or as a contamination of the isophotes of the underlying host galaxies. Indeed, that contamination should affect all the quasars to a greater or lesser extent (Andrei et al. 2015, 2012a). Another important purpose concerns the few radio-loud, ICRF2 defining quasars, upon which will be established the orientation of the GCRF and its continuity to the ICRF. For these objects asymmetries between optical and radio morphology would induce spurious centroids offsets (Camargo et al. 2011). On the same topic Zacharias \& Zacharias (2014) brought up to the community for the first time the issue of the radio-optical offsets. They used a set of ICRF extragalactic objects and compared their VLBI positions with the UCAC optical ones. Moreover a recent very accurate astrometric study dealing with a limited set of 12 extragalactic sources observed over a ten-year time span was carried out (Harris et al. 2016), which showed no significant photocentre motion on the 1-2 mas level, except for a BLAC object.

The morphological indexes determined in the LQAC series address departures of the light distribution of the quasar from a point-like stellar one (Coelho et al. 2012, 2014). They are briefly reviewed in the following.

First we notice that in the present fourth version of the LQAC, namely the LQAC-4, we introduced about $37.5 \%$ more quasars than in the preceding version (see Sect. 2). As expected, the newcomers are mostly fainter and farther objects than those for instance in the first version, the LQAC (Souchay et al. 2009). We notice, however, that this is not true for all the newly introduced objects, about one third of the new population corresponds to an enlargement of the sky coverage of the SDSS, keeping its standard observational procedures, and thus keeping the same magnitude and redshift distributions as before for this fraction.

Figures 17 and 18 bring the magnitude and redshift distribution of the 121461 new objects, for which a morphological index was sought. We note that the magnitude averages were, in the LQAC-2 (Souchay et al. 2012) and in the LQAC-3 (Souchay et al. 2015) respectively, 19.23 and 20.76, while the average redshifts were $z=1.40$ and $z=2.20$. For the new quasars introduced in the LQAC-4, although the magnitude distribution is quite similar to those of the LQAC-3, with an average value

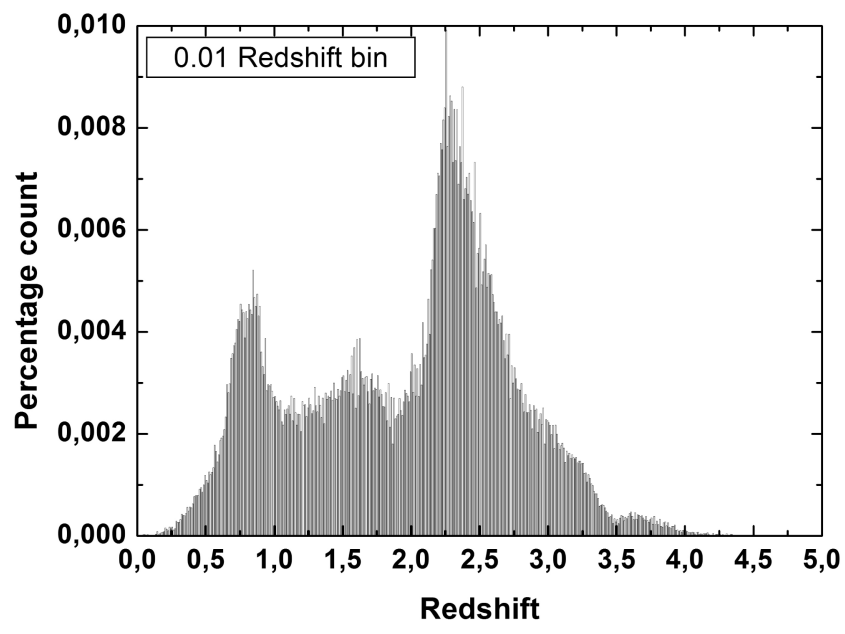

Fig. 18. Redshift distribution histogram for the 121461 new quasars in the LQAC4 for which DSS images and the morphological indexes were searched for and calculated. The percentage counts are obtained in 0.01 redshift bins. All redshift are spectroscopic. There are clearly two concentrations, inherited from the SDSS Main and BOSS populations.

at 20.87, the redshift distribution shows two distinctive peaks $(z \sim 0.8$ and $z \sim 2.3)$ and a sizeable plateau in-between (we note also a tiny peak at $z \sim 1.6$ ), as it represents a compound of the LQAC-2 and LQAC-3 distributions.

It is to be noted here that this redshift distribution is quite biased by the Sloan Digital Sky Survey target selection as it is a major contributor to the LQAC-4 catalogue. For example, the two peaks at $z \sim 0.8$ and $z \sim 1.6$ are known degeneracies of its selection process. SDSS has been divided into several phases (the current one being IV), each one reaching different goals. For instance, SDSS III focused on maximizing quasar discoveries at redshifts of between 2.15 and 3.5 (see more details in Pâris et al. 2017).

The morphological indexes, as in the former versions of the LQAC, were calculated using the approach of analysing the PSF of the quasar image in comparison with the local mean PSF of stars of similar magnitude. The elements of comparison are the parameters SHARP, SROUND, and GROUND, determined by IRAFA's DAOFIND on the Johnston $B, R$, and $I$ digitized plates of the DSS (Deep Space Survey). The morphological indexes of each quasar are given by (Andrei et al. 2012b):

$M_{P C}=\frac{\left|P_{Q}-\overline{P_{s}}\right|}{\sigma_{s}}$

where $M$ is the morphological index of quasar $Q$ for the PSF parameter $P$ in the colour $C$, given in comparison to the mean value from the stars $s$, and normalized by the stellar standard deviation $\sigma$.

Figure 19 depicts the distribution of the morphological indexes. Since the bulk of these new sources comes from the SDSS BOSS population, as was the case for the LQAC-3, there is no difference between the behaviour on either DSS band. The redshift value $z=2.3$, which represents the peak of the present distribution, corresponds to so-called quasar golden age, when the number and brightness of quasars seemed to be maximal. It also corresponds to the presence of large host-galaxies rich in both dust and gas, and harbouring a high rate of young stars. The linear correlation of the morphological indexes distribution of new sources coming from the DR12Q to the one from the 


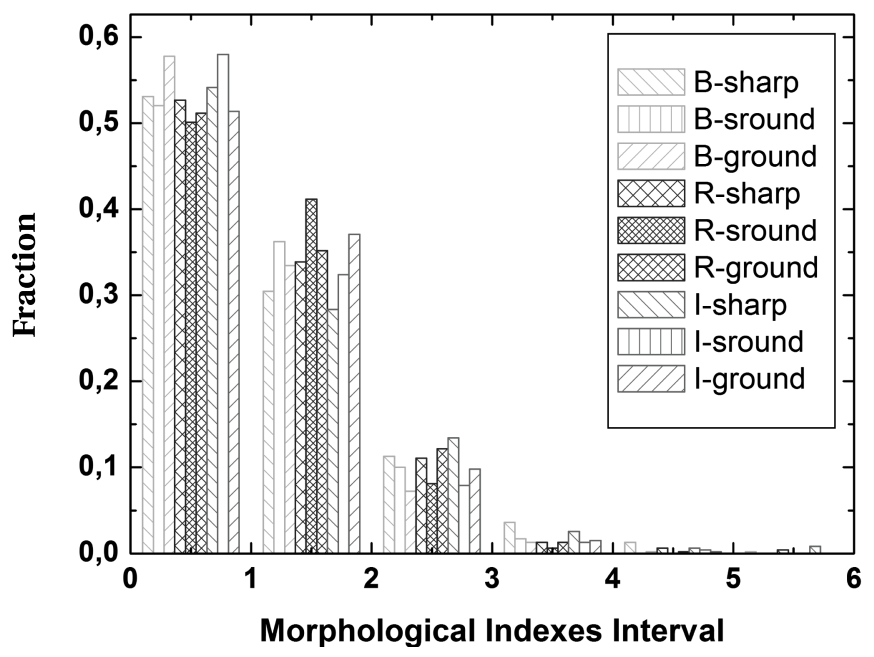

Fig. 19. Histograms of the morphological indexes for the new quasars in the LQAC4. The binsize in abscissa is reduced for the sake of clarity. Each bin refers to the integer range in which it is located (e.g. $[0,1])$. The fractions in ordinate refers to the total number of sources for which each of the nine morphological indexes were determined. Letters $B, R$, and $I$ refer to the three colours of DSS plates, blue, red, and infrared. The mnemonic after the letters refer to the IRAFA's DAOFIND morphological index, respectively sharp, sround, and ground.

sources compiled in the LQAC-3 is 0.996 and asserts the reality of the distribution.

As in the previous version LQAC-3 (Souchay et al. 2015), although most of the quasars show up as point-like objects, about $10 \%$ have morphological indexes larger than two, and about $2 \%$ have morphological indexes larger than three. Thus the behaviour of the different indexes is approximately the same as in the LQAC-3. The modal value for the three indexes on the three colours is about one. This means that, although the large majority of objects are statistically stellar-like, they show up as clearly different morphological entities than stars. We note that no dependence of the morphological indexes with redshift was found.

\section{Celestial Reference Frame coordinates with Gaia DR1 input}

The main objective of the LQAC-4, as its acronym justifies it, is to give to each quasar the a priori best equatorial coordinates according to the quality of the source of these coordinates. On this topic, three potentially different kinds of sources can be found. The first one is the individual quasars catalogue itself (flags A to I) in which the original coordinates have been picked up. The second one is the LQRF (Andrei et al. 2009), i.e. a catalogue improving the global accuracy of the positions of the quasars thanks to a set of algorithms respecting an alignment to the ICRF and the use of intermediary all-sky catalogues as the UCAC2 (Zacharias et al. 2004). The third source is the Gaia DR 1 catalogue. From these three kinds of sources, a general rule is adopted for the selection of the a priori best coordinates.

The first step of this rule consists of selecting, when available, the VLBI coordinates from the ICRF catalogue. It concerns only the 3414 sources that constitute the whole ICRF with flag $\mathrm{A}$ in the LQAC. As they constitute by definition the primary reference frame with a set of positions with an optimal astrometric quality (Fey et al. 2015) the coordinates of the ICRF2 are privileged with respect to those of the Gaia DR1 or of the LQRF even when they are available. The second step of this

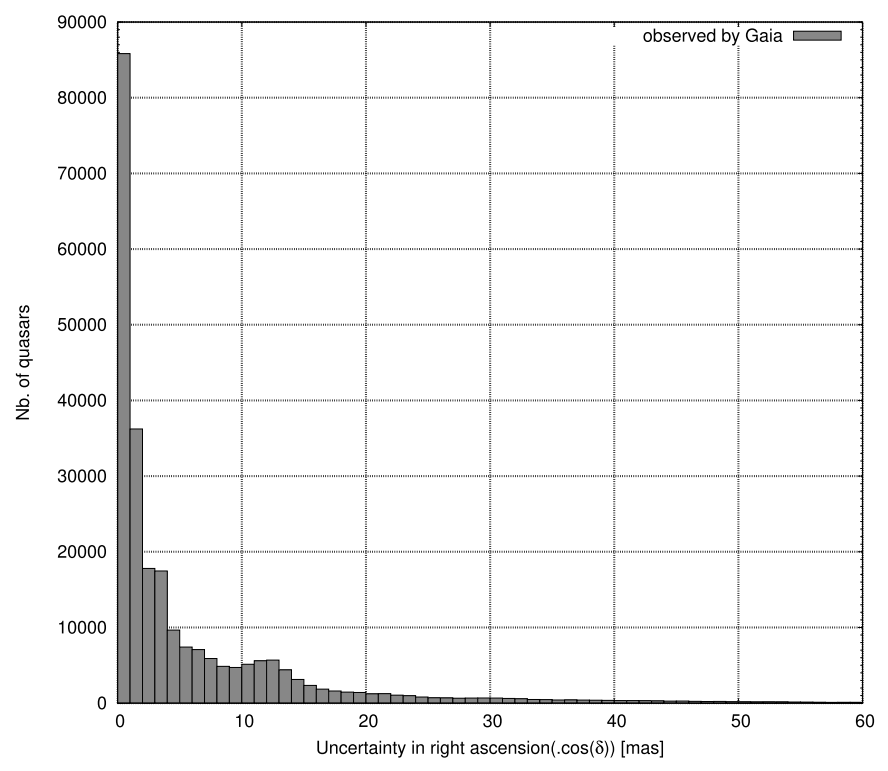

Fig. 20. Histogram of uncertainty in right ascension of the 249071 quasars of the LQAC-4 cross-matched with Gaia. Values are taken directly from the Gaia DR1

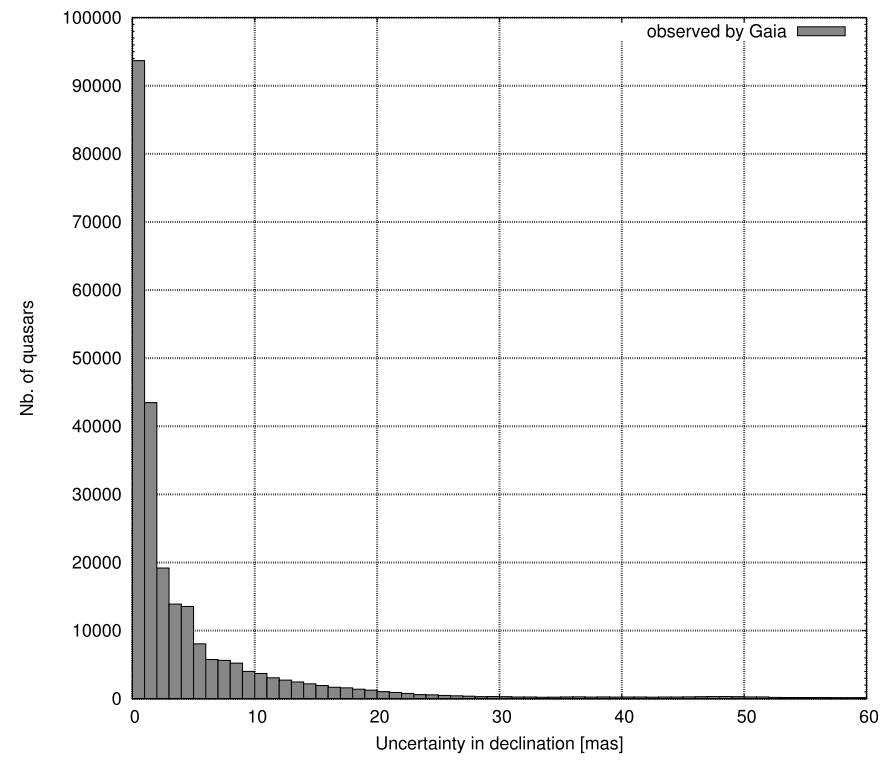

Fig. 21. Histogram of uncertainty in declination of the 249071 quasars of the LQAC-4 cross-matched with Gaia. Values are taken directly from the Gaia DR1

rule consists of selecting, when available, the Gaia DR1 coordinates. As mentioned above and illustrated in Table 1, these GDR1 coordinates are available for 249071 quasars of the LQAC-4, corresponding to $56.13 \%$ of the whole LQAC-4 population. Then in the second step, we concentrate on the population without DR1 counterpart: for this population, we search for the possibility that LQRF coordinates are available. In the positive case we catch right ascension and declination of the quasar as given by the LQRF. In the negative case, we have no other solution than picking up the celestial coordinates from the original catalogue.

Finally, the counts for optimal positions are the following ones: 249071 sets of coordinates ( $\alpha$ and $\delta$ ) come from the Gaia DR1 data, 119924 sets from the LQRF and 74730 sets are extracted from the individual quasars catalogues. In 
Figs. 20 and 21 we show the histograms of the uncertainties respectively in $\alpha \cdot \cos \delta$ and $\delta$ for quasars for which the coordinates have been extracted from the Gaia-DR1. These uncertainties come from the GDR1 catalogue itself. We remark that, for the large majority of objects, the uncertainty is smaller than 20 mas which constitutes a major improvement on the standard coordinates difference between GDR1 and the various groundbased catalogues, as displayed in Table 3. Moreover, other improvements are expected from the future releases of Gaia, with coordinates values based on a significantly larger set of measurements of the quasars detected.

\section{Conclusion}

In this paper we have constructed an updated version of the Large Quasar Astrometric Catalogue, namely the LQAC-4, by adding exclusively new quasars coming from the DR12Q release of the SDSS (Pâris et al. 2017). The total number of recorded quasars in our compilation reaches 443725 objects, that constitutes a $37.82 \%$ increase with respect to the 321957 objects of the LQAC-3 compilation (Souchay et al. 2015).

A new and important element of this paper is the crossidentification of the LQAC-4 objects with the Gaia DR1 catalogue, which enables us to fully reckon Gaia objects as already recorded quasars. Thus, with a $1^{\prime \prime}$ search radius for cross-identification, 249071 Gaia objects were recognized as quasars, that represents $56.13 \%$ of the whole population in the LQAC-4. Thanks to this cross-identification, we have calculated the standard deviation in right ascension and declination for each individual catalogue participating in the LQAC-4 compilation, following a hierarchy among the catalogues in terms of astrometric precision. In particular, we have pointed out a systematic significant offset of the coordinates given by the SDSS with respect to the Gaia DR1 for a well delimited zone of the SDSS survey.

In addition, we have carried out a study of the global rotations between the celestial frame associated with each individual catalogue and the celestial frame associated with the Gaia catalogue defined implicitly through the equatorial coordinates of the objects. Moreover we have adopted a new method of calculation of the absolute magnitudes of the sample of new quasars coming from the DR12Q. This new method, set up by Coelho et al. (2017) has been applied for the 121768 new quasars included in the LQAC-4, to determine the absolute magnitudes in the five SDSS bands, respectively $M_{u}, M_{g}, M_{r}, M_{i}$ and $M_{z}$. We have also determined the morphological indexes for the additional quasars above, by adopting the same algorithms based on IRAF tools as in previous up-dates of the LQAC.

Finally, as a fundamental task of our astrometric catalogue, we give for each quasar of the compilation its best estimate of equatorial coordinates, based preferentially on ICRF values (for 3414 objects), then on Gaia DR1 values (for the 249071 cross-matched objects) then on the LQRF calculations (119924 objects), and for the remaining cases (74 730 objects) on the a priori best catalogue of the compilation, in an astrometrical point of view. We think that the LQAC-4 will represent to the astronomical community, as did its predecessors, a fundamental tool gathering the very large majority of recorded quasars with various information, in particular the data coming from the Gaia DR1.

Acknowledgements. We thank C. Babusiaux (Observatoire de Paris/GEPI) for useful advice on the use of the Gaia DR1 catalogue, S. Derriere (CDS, Strasbourg) for his help when using the VO tools, T. Carlucci (Observatoire de
Paris/SYRTE) for the maintenance of the LQAC data servers and S. Lambert \& J.-Y. Richard (Observatoire de Paris/SYRTE) for useful advice on the statistical approach used in these studies. A. H. Andrei thanks the Observatoire de Paris/SYRTE and the $\mathrm{CNPq} / \mathrm{Brazil}$ for grants received to join the project. This work has made use of data from the European Space Agency (ESA) mission Gaia (https: //www. cosmos.esa.int/gaia), processed by the Gaia Data Processing and Analysis Consortium (DPAC, https://www.cosmos.esa.int/web/ gaia/dpac/consortium). Funding for the DPAC has been provided by national institutions, in particular the institutions participating in the Gaia Multilateral Agreement. This work has made use of data from the SDSS-III data catalogue. Funding for SDSS-III has been provided by the Alfred P. Sloan Foundation, the Participating Institutions, the National Science Foundation, and the US Department of Energy Office of Science. The SDSS-III web site is http://www. sdss3.org/.SDSS-III is managed by the Astrophysical Research Consortium for the Participating Institutions of the SDSS-III Collaboration including the University of Arizona, the Brazilian Participation Group, Brookhaven National Laboratory, Carnegie Mellon University, University of Florida, the French Participation Group, the German Participation Group, Harvard University, the Instituto de Astrofisica de Canarias, the Michigan State/Notre Dame/JINA Participation Group, Johns Hopkins University, Lawrence Berkeley National Laboratory, Max Planck Institute for Astrophysics, Max Planck Institute for Extraterrestrial Physics, New Mexico State University, New York University, Ohio State University, Pennsylvania State University, University of Portsmouth, Princeton University, the Spanish Participation Group, University of Tokyo, University of Utah, Vanderbilt University, University of Virginia, University of Washington, and Yale University.

\section{References}

Andrei, A. H., Souchay, J., Zacharias, N., et al. 2009, A\&A, 505, 385

Andrei, A. H., Anton, S., Barache, C., et al. 2012a, Mem. Soc. Astron. It., 83 930

Andrei, A. H., Anton, S., Barache, C., et al. 2012b, in SF2A-2012: Proc. of the Annual meeting of the French Society of Astronomy and Astrophysics, eds. S. Boissier, P. de Laverny, N. Nardetto, et al., 61

Andrei, A. H., Souchay, J., Martins, R. V., et al. 2012c, in IAU Joint Discussion, 7, 31

Andrei, A., Coelho, B., \& Anton, S. 2015, in Journées Systèmes de référence spatio-temporels 2014, eds. Z. Malkin \& N. Capitaine

Arias, E. F., Charlot, P., Feissel, M., \& Lestrade, J.-F. 1995, A\&A, 303, 604

Bachchan, R. K., Hobbs, D., \& Lindegren, L. 2016, A\&A, 589, A71

Camargo, J. I. B., Andrei, A. H., Assafin, M., Vieira-Martins, R., \& da Silva Neto D. N. 2011, A\&A, 532, A115

Claeskens, J.-F., Smette, A., Vandenbulcke, L., \& Surdej, J. 2006, MNRAS, 367, 879

Coelho, B., Andrei, A., Anton, S., et al. 2012, in Journées Systèmes de Référence Spatio-temporels 2011, eds. H. Schuh, S. Boehm, T. Nilsson, \& N. Capitaine, 13

Coelho, B., Andrei, A. H., \& Antón, S. 2014, Rev. Mex. Astron. Astrofis. Conf. Ser., 44, 191

Coelho, B., Andrei, A., \& Anton, S. 2017, A\&A, submitted

Croom, S. M., Smith, R. J., Boyle, B. J., et al. 2004, MNRAS, 349, 1397

Dawson, K. S., Schlegel, D. J., Ahn, C. P., et al. 2013, AJ, 145, 10

Dawson, K. S., Kneib, J.-P., Percival, W. J., et al. 2016, AJ, 151, 44

de Veny, J. B., Osborn, W. H., \& Janes, K. 1971, PASP, 83, 611

Eisenstein, D. J., Weinberg, D. H., Agol, E., et al. 2011, AJ, 142, 72

Fey, A. L., Gordon, D., Jacobs, C. S., et al. 2015, AJ, 150, 58

Finet, F., \& Surdej, J. 2016, A\&A, 590, A42

Gaia Collaboration (Brown, A. G. A., et al.) 2016a, A\&A, 595, A2

Gaia Collaboration (Prusti, T., et al.) 2016b, A\&A, 595, A1

Gordon, K. D., Clayton, G. C., Misselt, K. A., Landolt, A. U., \& Wolff, M. J 2003, ApJ, 594, 279

Gunn, J. E., Siegmund, W. A., Mannery, E. J., et al. 2006, AJ, 131, 2332

Harris, H. C., Dahn, C. C., Zacharias, N., et al. 2016, AJ, 152, 118

Hobbs, D., Lindegren, L., Bastian, U., et al. 2017, Gaia DR1 Documentation Chapter 3: Astrometry

Hogg, D. W. 2000, ArXiv e-prints [arxiv:astro-ph/9905116v4]

Hopkins, P. F., Strauss, M. A., Hall, P. B., et al. 2004, AJ, 128, 1112

Jordi, C., Gebran, M., Carrasco, J. M., et al. 2010, A\&A, 523, A48

Kovalev, Y. Y., Petrov, L., \& Plavin, A. V. 2017, A\&A, 598, L1

Krawczyk, C. M., Richards, G. T., Gallagher, S. C., et al. 2015, AJ, 149, 203

Lindegren, L., Lammers, U., Bastian, U., et al. 2016, A\&A, 595, A4

Lynds, R. 1971, ApJ, 164, L73

Meiksin, A. 2006, MNRAS, 365, 807

Michalik, D., \& Lindegren, L. 2016, A\&A, 586, A26

Mignard, F., Klioner, S., Lindegren, L., et al. 2016, A\&A, 595, A5 


\section{Gattano et al.: LQAC-4: Fourth release of the Large Quasar Astrometric Catalogue}

Myers, A. D., Palanque-Delabrouille, N., Prakash, A., et al. 2015, ApJS, 221, 27

Pâris, I., Petitjean, P., Ross, N. P., et al. 2017, A\&A, 597, A79

Petrov, L., \& Kovalev, Y. Y. 2017a, MNRAS, 471, 3775

Petrov, L., \& Kovalev, Y. Y. 2017b, MNRAS, 467, L71

Rauch, M. 1998, ARA\&A, 36, 267

Ross, N. P., Myers, A. D., Sheldon, E. S., et al. 2012, ApJS, 199, 3

Schlegel, D. J., Finkbeiner, D. P., \& Davis, M. 1998, ApJ, 500, 525

Schneider, D. P., Richards, G. T., Hall, P. B., et al. 2010, AJ, 139, 2360
Schuh, H., \& Behrend, D. 2012, J. Geodyn., 61, 68

Secrest, N. J., Dudik, R. P., Dorland, B. N., et al. 2015, ApJS, 221, 12

Souchay, J., Andrei, A. H., Barache, C., et al. 2009, A\&A, 494, 799

Souchay, J., Andrei, A. H., Barache, C., et al. 2012, A\&A, 537, A99

Souchay, J., Andrei, A. H., Barache, C., et al. 2015, A\&A, 583, A75

Véron-Cetty, M.-P., \& Véron, P. 2010, A\&A, 518, A10

York, D. G., Adelman, J., Anderson, Jr. J. E., et al. 2000, AJ, 120, 1579

Zacharias, N., \& Zacharias, M. I. 2014, AJ, 147, 95

Zacharias, N., Urban, S. E., Zacharias, M. I., et al. 2004, AJ, 127, 3043 